\title{
Design and Analysis of Two Stage Op-Amp for Bio-Medical
}

\section{Application}

\author{
Wang Jin1), Praful Ranjan2)
}

\begin{abstract}
Op Amps are the main building block in analog IC designing. Most of the real world signals are mostly analog and they need to be converted into digital signals to analyze and process them. So, analog circuits are of significant importance for us. We have been able to implement a Two Stage Op-Amp using Cadence Tools. We successfully achieved the specifications that we took as a reference in our design, and accordingly were able to design a high gain Op-Amp with low power dissipation. We started with our work keeping in mind the biomedical applications like ECG and EMG. After critically analyzing the design, gain of $72.54 \mathrm{db}$ and power consumption of $1.02 \mathrm{~mW}$ is achieved with CMMR 60 and gain bandwidth product 16.653 .
\end{abstract}

Keywords : Op-amp, Cadence virtuoso, Gain, CMRR, Bio-Medical application

\section{Introduction}

Operational Amplifier (Op-Amp) is the important basic building block of any analog circuit. The conventional operational amplifier has a high voltage gain differential input stage with a second stage having high voltage gain with a last stage called buffer stage. Buffer stage provides stumpy voltage gain and large current gain. The first two stages are responsible for providing the overall voltage gain of the operational amplifier circuit. This operational amplifier circuit sometimes called as the Widlar architecture [1-2]. Compensating capacitor is placed between the output of the first stage and the input of the second stage. CMOS (Complementary metal-oxide-semiconductor) circuit is basic building block for designing operational amplifier because CMOS circuits have various advantages over BJT (Bipolar Junction Transistor) including power consumption and noise reduction. The CMOS required less fabrication steps than BJT.

We mainly needed to concentrate on designing a low power CMOS amplifier that would need low supply voltages, and will still have the capability to amplify very weak signals to

Received(May 31, 2017), Review Result(1st: June 15, 2017, 2nd: July 28, 2017), Accepted(August 10, 2017)

${ }^{1}$ Yangzhou University, 88 South University Ave., Yangzhou, Jiangsu 225009, P. R. China email: jinwang@yzu.edu.cn

${ }^{2}$ THDC-Institute of Hydropower Engineering \& Technology, Tehri, India email: prafulmirchi@gmail.com 
make them suitably strong for further processing [3-5]. Our main aim is to keep biomedical applications in mind while designing the Op-Amp. In such applications, the amplifier (along with other necessary circuits) comes in direct contact with the human body. It is mandatory to keep power requirements and supplied voltages under control so that the user doesn't get an electric shock. We have tried to address this issue to some extent in our Op-Amp.

\section{Block Diagram of the Proposed Design}

The first stage of an op-amp is a differential amplifier stage. Differential Amplifier having two inputs and the difference of these two input will be amplified [6-9]. The first stage provides the maximum gain of an op-amp. The second stage is a common source stage. The compensation capacitance (CC) is placed between first stage and second stage. The CC provides the stability to the op-amp. The CC increase the phase margin of the design and for a stable system the phase margin is should be greater than 450 . The second stage increases the gain of the op-amp and converts the second stage input voltage into the current.

The second stage is nothing more than the current sink inverter [10-12]. The second stage is a common source stage which increases the DC voltage gain and improve the output signal swing for a given power supply voltage. The second stage is followed by a buffer stage, which provides a lower the output resistance and maintains a large voltage swing. The buffer stage also provides better slew rate (SR) of an Op-Amp. It is not necessary all applications require low output impedance. If the Op-Amp is intended to drive a small purely capacitive load, which is the case in many switched capacitor or data conversion applications, the output buffer is not used. The Biasing current is used to provide to keep all the transistors in saturation region [13-15]. The purpose of the compensation circuit or compensation capacitance is to reduce the overall gain at higher frequencies and provides stability when negative feedback is applied to the Op-Amp.

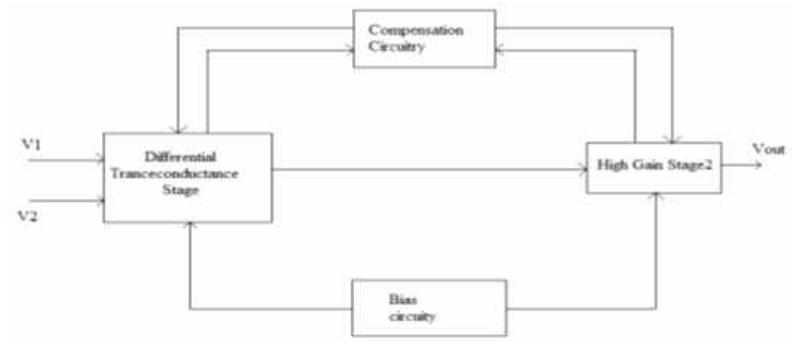

[Fig. 1] Block Diagram of Two Stage Differential Amplifier 


\section{Simulation Set Up}

Cadence Virtuoso 6.1 .5 is used as a simulation tool for simulating Op-amp design. Advanced features like Cadence Virtuoso Schematic Editor for providing high speed and easy design methods, Cadence Virtuoso Layout Suite for speeding up the physical layout of the design, Cadence Virtuoso Visualization and Analysis for efficiently analyzing the performance of the design and Cadence Assura Physical Verification for reducing overall verification time, because a quick and instinctive debug capability is incorporated within the Virtuoso custom design environment. It easily compares, repair, remove and distinguish errors.

\section{Performance Parameters of High Gain Amplifier}

\subsection{Open Loop Gain}

The ratio of the output voltage and differential input voltage is called open loop gain of op-amp. Since the output signal is much larger than the input signal, therefore it is termed as large signal voltage gain. It may be expressed in decibels using the following relation,

$$
\mathrm{AV}(\mathrm{dB})=20 \log _{10}\left(\mathrm{~V}_{\text {out }} / \mathrm{V}_{\mathrm{DM}}\right)
$$

where, VDM is the differential input voltage and Vout is the output voltage.

\subsection{Miller Effect}

The effective input impedance of an amplifier depends on the impedance connected from input to output of the amplifier. The perceptible scaling of this impedance often dominates the input impedance and frequency response of the amplifier. This type of phenomena commonly known as the Miller Effect. The virtually increased input capacitance due to the Miller effect is given by:

$$
\mathrm{C}_{\mathrm{M}}=\mathrm{C}\left(1+\mathrm{A}_{\mathrm{V}}\right)
$$

where, $A_{V}$ is the gain of the amplifier and $C$ is the feedback capacitance.

\subsection{Gain Bandwidth Product}


For an amplifier the gain bandwidth product designated as GB is the product of the amplifier's gain and the bandwidth. For devices such as Op-Amps that are designed to have a simple one-pole frequency response. In case of that type of design the GB is nearly independent of the gain at which it is measured. So in this kind of devices the gain bandwidth product will also be equal to the unity gain bandwidth of the amplifier.

\subsection{Slew Rate}

Slew rate $(\mathrm{SR})$ is the rate of change of output voltage caused by changed in input. Its units are $\mathrm{V} / \mathrm{ms}$ or $\mathrm{V} / \mathrm{us}$.

Internal compensation capacitor $\mathrm{CC}$ is the primary factor controlling slew rate in most Op-Amps, which makes the Op-Amp unity gain stable. Charging and discharging of the compensation capacitor CC limits the voltage change in the second stage. The maximum rate of change is when either side of the differential pair is conducting 2IE. All Op-Amps do not have compensation capacitors. In Op-Amps without internal compensation capacitors, the slew rate is determined by internal Op-Amp parasitic capacitances.

\subsection{Gain Margin and Phase Margin}

The amount of gain increase or decrease required to make the loop gain unity at the frequency $\omega \mathrm{gm}$ (phase crossover frequency, where the phase angle is $-180^{\circ}$ )is called the gain margin. The gain margin is $1 / \mathrm{f}$ if $\mathrm{f}$ is the gain at the $-180^{\circ}$ phase frequency.

The difference between the phase of the response and $-180^{\circ}$ is called the phase margin when the loop gain is 1.0 (or $0 \mathrm{~dB}$ ). The unity-gain frequency or gain crossover frequency is that frequency wpm at which the magnitude is 1.0 .

\section{Designing of Op-Amp}

The Op-Amp design process mainly involves the two major steps. First one is called Design conception while another one is termed as Design optimization. An architecture is proposed to meet the given specifications in design conception. This step is usually done to calculate the design values by hand calculations which is necessary for choices that must be made. The "first-cut" design is taken \& verified for the specifications and then optimized in the second step of the design optimization. The optimization can include different environmental influences 
or process variations and is usually done by using Computer simulation.

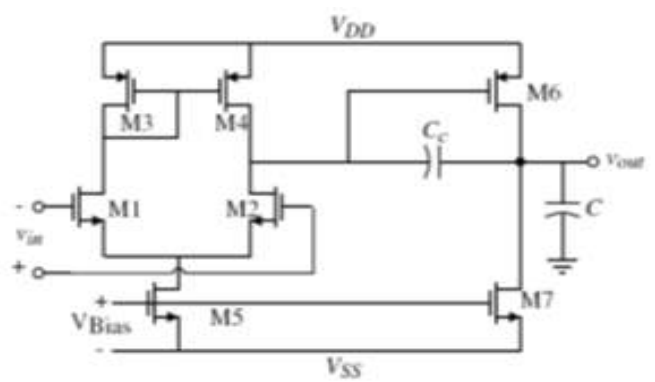

[Fig. 2] Architecture of 2-stage Op-Amp

Model or Device Parameters:

$\mathrm{K}_{\mathrm{p}}=\mu_{\mathrm{p}} \mathrm{C}_{\mathrm{ox}}=60 \mu \mathrm{A} / \mathrm{V}^{2}$

$\mathrm{K}_{\mathrm{n}}=\mu_{\mathrm{p}} \mathrm{C}_{\mathrm{ox}}=300 \mu \mathrm{A} / \mathrm{V}^{2}$

Threshold Voltage $\mathrm{V}_{\mathrm{TH}(\mathrm{n})}=0.61 \mathrm{~V}, \mathrm{~V}_{\mathrm{TH}(\mathrm{p})}=0.46 \mathrm{~V}$.

First Stage (Differential Amplifier) Design Values:

The values of the $(\mathrm{W} / \mathrm{L})$ were calculated as follows:

$\mathrm{S}_{1,2}=28, \mathrm{~S}_{3,4}=84, \mathrm{~S}_{5,8}=4$.

DC current $\mathrm{I}_{\mathrm{C}}=50 \mu \mathrm{A}, \mathrm{V}_{\mathrm{DD}}=1.8 \mathrm{~V}, \mathrm{~V}_{\mathrm{SS}}=-1.8 \mathrm{~V}, \mathrm{C}_{\mathrm{L}}=10 \mathrm{pF}$.

\subsection{Circuits and Simulated Outputs of First Stage (Differential Amplifier)}

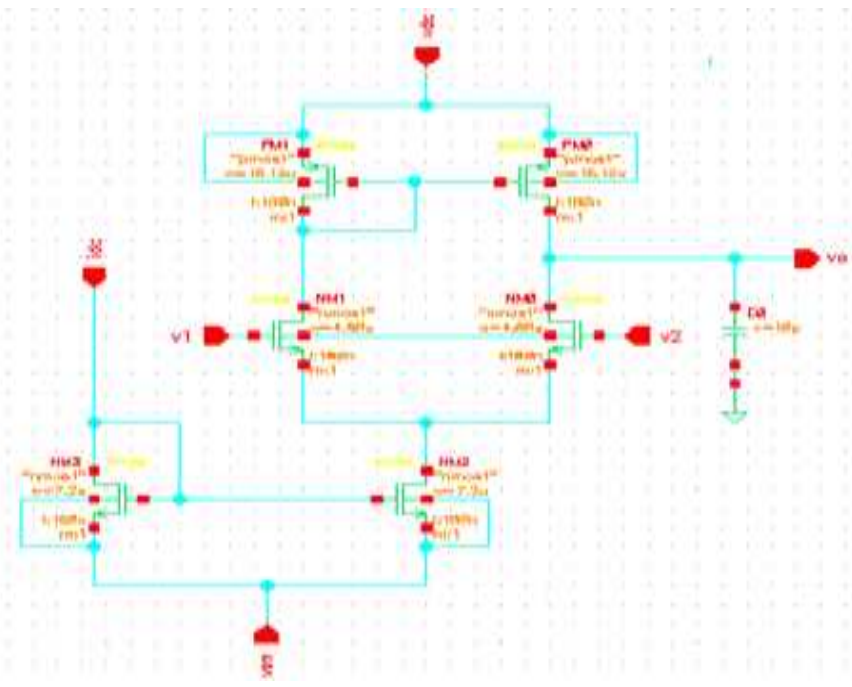

[Fig. 3] Schematic of First Stage Op-Amp 


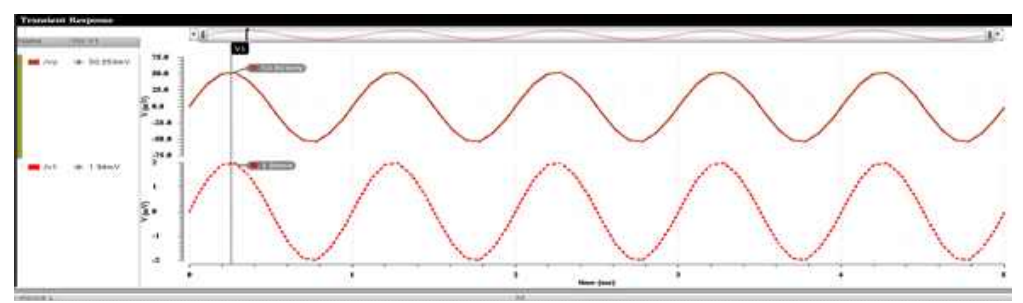

[Fig. 4] Transient Response of First Stage Op-Amp

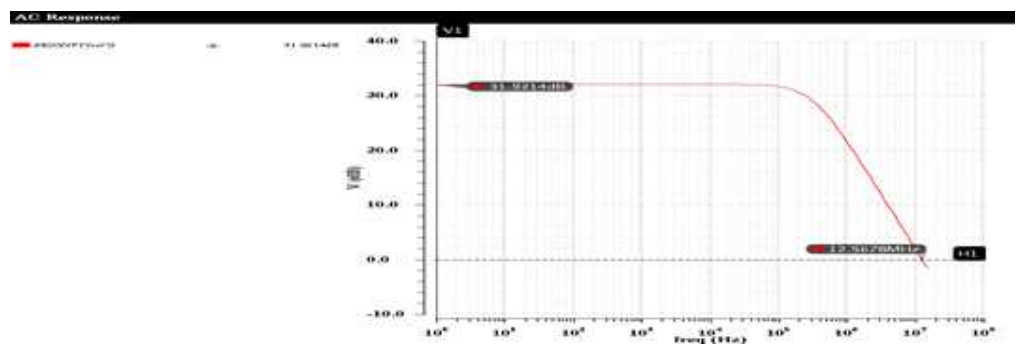

[Fig. 5] A.C Response of First Stage of Op-Amp

Second Stage Design Values:

The aspect ratio $(\mathrm{W} / \mathrm{L})$ values for different transistors were calculated as follows:

$\mathrm{S}_{1,2}=6, \mathrm{~S}_{3,4}=8, \mathrm{~S}_{5,8}=8, \mathrm{~S}_{6}=154, \mathrm{~S}_{7}=102$.

DC current $\mathrm{I}_{\mathrm{C}}=15 \mu \mathrm{m}, \mathrm{V}_{\mathrm{DD}}=1.8 \mathrm{~V}, \mathrm{~V}_{\mathrm{SS}}=-1.8 \mathrm{~V}, \mathrm{C}_{\mathrm{C}}=2.2 \mathrm{pF}, \mathrm{C}_{\mathrm{L}}=10 \mathrm{pF}$.

\subsection{Circuits and Simulated Outputs after addition of Second Stage}

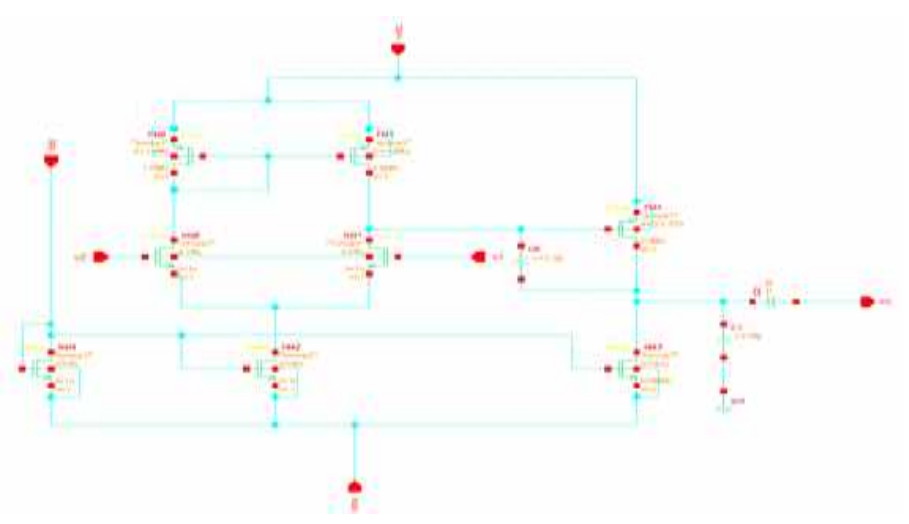

[Fig. 6] Two Stage Op-Amp Schematic 


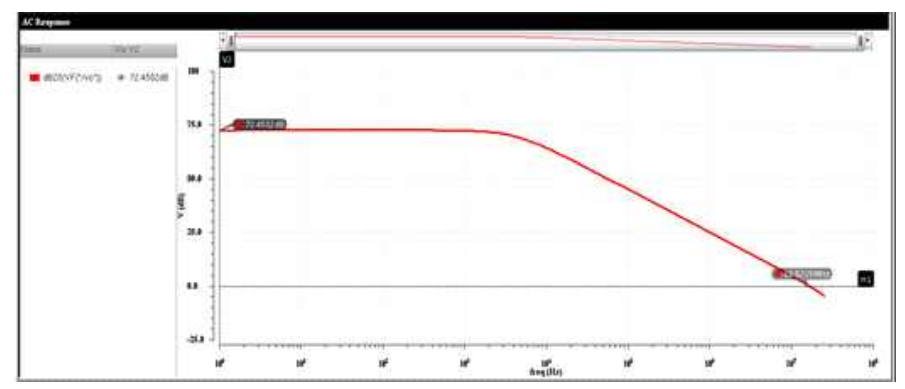

[Fig. 7] AC dB 20 Plot for Gain and Bandwidth

\section{Result and Conclusion}

Design specification values obtained after simulation are tabulated in Table 1 . The obtained results are further compared with the reference papers and shown in table 2.

[Table 1] Values obtained after simulation

\begin{tabular}{|c|c|c|c|}
\hline $\begin{array}{c}\text { S. } \\
\text { No. }\end{array}$ & Specifications & Proposed Values & Obtained Values \\
\hline 1 & Technology & 180 nanometres & Used \\
\hline 2 & Power Supply $\left(\mathrm{V}_{\mathrm{dd}}\right)$ & $1.8 \mathrm{~V}$ & $1.8 \mathrm{~V}$ \\
\hline 3 & Load Capacitance $\left(\mathrm{C}_{\mathrm{L}}\right)$ & $10 \mathrm{pF}$ & $10 \mathrm{pF}$ \\
\hline 4 & Coupling Capacitance $(\mathrm{C}-\mathrm{c})$ & $2.2 \mathrm{pF}$ & $2.2 \mathrm{pF}$ \\
\hline 5 & Power dissipation $\left(\mathrm{P}_{\mathrm{D}}\right)$ & $<=2 \mathrm{~mW}$ & $1.003 \mathrm{~mW}$ \\
\hline 6 & Differential DC gain $(\mathrm{Av})$ & $>=60 \mathrm{~dB}$ & $72.54 \mathrm{~dB}$ \\
\hline 7 & Unity Gain Bandwidth & $>10 \mathrm{Mega} \mathrm{hertz}$ & $16.653 \mathrm{MHz}$ \\
\hline 8 & Phase Margin $(\mathrm{PM})$ & $>=60 \mathrm{deg}$ & $61.417 \mathrm{deg}$ \\
\hline 9 & Common Mode Rejection $(\mathrm{CMRR})$ & $>=60 \mathrm{~dB}$ & $60 \mathrm{~dB}$ \\
\hline
\end{tabular}

[Table 2] Comparison of Obtained Results with Reference Papers

\begin{tabular}{|c|c|c|c|c|c|c|c|c|}
\hline \multirow{2}{*}{ Parameters } & \multicolumn{6}{|c|}{ References } & \multirow{2}{*}{$\begin{array}{c}\text { Results of } \\
\text { Proposed Design }\end{array}$} \\
\cline { 2 - 10 } & {$[\mathbf{6}]$} & {$[9]$} & {$[\mathbf{1 1}]$} & {$[\mathbf{1 2}]$} & {$[\mathbf{1 3}]$} & {$[\mathbf{1 4}]$} & {$[\mathbf{1 6}]$} & \\
\hline Gain (dB) & 36.747 & 67 & 78 & 71 & 51 & 50 & 70 & 72.54 \\
\hline Gain BW Product (MHz) & 16 & 12 & 11 & 5 & - & 10 & 14 & 16.653 \\
\hline Power Dissipation (mW) & 0.804 & - & - & $<2$ & - & 2.4 & 2.1 & 1.003 \\
\hline CMRR (dB) & - & 78 & 60 & - & 64 & - & - & 60 \\
\hline
\end{tabular}




\begin{tabular}{|c|c|c|c|c|c|c|c|c|}
\hline Phase Margin (deg) & 48.1 & 59 & 85.85 & - & - & 175 & 65 & 61.417 \\
\hline Power Supply (V) & - & - & 5 & 2.5 & 2.3 & - & - & 1.8 \\
\hline Technology (nm) & 180 & - & $\begin{array}{c}\text { tsmc } \\
350\end{array}$ & 180 & 90 & 90 & 180 & 180 \\
\hline
\end{tabular}

\section{References}

[1] Douglas R. Holberg and Philip E. Allen, "CMOS Analog Circuit Design, Second Edition", Oxford University Press, 2002.

[2] Behzad Razavi, "Design of Analog CMOS Integrated Circuits", McGraw-Hill, 2002.

[3] Sung-Mo Kang, Yusuf Leblebici, "CMOS Digital Integrated Circuits, Analysis and design”, Tata McGraw-Hill Edition 2003.

[4] P.R. Gray, P.J. Hurst, S.H. Lewis and R.G. Meyer, "Analysis and Design of Analog Integrated Circuits", Fourth Edition, John Wiley \&Sons, Inc., 2001.

[5] A.S Sedra and K.C.Smith ,"Microelectronics Circuits Theory and Applications", Fifth Edition. Oxford University Press, 2009.

[6] Sayan Bandyopadhyay, Deep Mukherjee "Design Of Two Stage CMOS Op-Amp in 180nm Technology With Low Power and High CMRR", Int. Journal of Recent Trends in Engineering \& Technology, Vol. 11, June 2014.

[7] Ajay Dadoria, C.M Roy et.al ,"CMOS Current Mirrors Comparison", International Journal of Advanced Research in Computer Science and Software Engineering April - 2013, pp. 625-630.

[8] Rajkumar S. Parihar \& Anu Gupta, "Design of a Fully Differential Two-Stage CMOS Op-Amp for High Gain, High Bandwidth Applications", May 2006.

[9] G. Palmisano, G. Palumbo and S. Pennisi, "Design Procedure for Two-Stage CMOS Transconductance Op-Amps: A Tutorial", Dipartimento Elettrico Elettronico e Sistemistico, Universita' di Catania, Viale Andrea Doria, 6 I-95125 Catania, Italy, September 12, 2000.

[10] Anshu Gupta, U.B.S. Chandrawat, D.K. Mishra, R. Khatri, "A Two Stage and Three Stage CMOS OPAMP with Fast Settling, High DC Gain and Low Power Designed in 180nm Technology", International Conference on Computer Information Systems and Industrial Management Applications, 2010.

[11] Amana Yadav "Design of Two-Stage CMOS Op-Amp and Analyze the Effect of Scaling" IJERA Vol. 2, Issue 5, September- October 2012, pp.647-654.

[12] M.Vinoth Kumar "Design of Mixed-Signal Integrator for RF Amplifier", International Journal of Computer Applications (0975 - 8887) International Conference on Innovations In Intelligent Instrumentation, Optimization And Signal Processing "ICIIIOSP-2013".

[13] Praveen Kumar Pandey, Aman Jain, "Design of Low-Voltage Op-Amp (700 mV)", International Journal of Engineering and Innovative Technology (IJEIT) Volume 3, Issue 1, July 2013. 
[14] O. M. Saravanakumar, N. Kaleeswari, K. Rajendran, "Design and Analysis of Two-Stage Operational Transconductance Amplifier (OTA) using Cadence tool", International Journal of Emerging Technology and Advanced Engineering, Volume 4, Issue 4, April 2014.

[15] Anand Kumar Singh, Anuradha, Dr. Vijay Nath, "Design and Performance analysis of Low power CMOS Op-Amp", International Journal of Engineering Sciences \& Research, September 2013.

[16] B.Krishna, D. Arunkumar, "A Novel Design of Low-Power Two Stage Cascode Op-Amp", International Journal of Advanced Research in Computer Science and Software Engineering, Volume 4, Issue 5, May 2014. 